\title{
COMPARISON OF OPTICAL PROPERTIES OF CdTe/CdMnTe QUANTUM WELLS GROWN BY MOLECULAR BEAM AND ATOMIC LAYER EPITAXY
}

M. Godlewski, K. Kopalko, T. Wojtowicz, G. Karczewski, J. Kossut

Institute of Physics, Polish Academy of Sciences

Al. Lotników 32/46, 02-668 Warsaw, Poland

J.P. Bergman and B. Monemar

Dept. of Physics and Meas. Technol., Linköping Univ., 58183 Linköping, Sweden

Optical properties of a series of $\mathrm{CdTe} / \mathrm{CdMnTe}$ multi quantum well structures grown with MBE and ALE (CdTe quantum wells only) methods are compared. Based on the results of the photoluminescence experiments we conclude that the ALE growth leads to a different lateral scale of quantum well width fluctuations, which results in different exciton properties in two multi quantum well systems studied. In the wells grown with ALE method excitons are less localized. They can migrate in a quantum well plane between quantum well regions varying in thickness by 1 monolayer.

PACS numbers: 71.35.+z, 78.47.+p, 78.55.Et

\section{Introduction}

Atomic layer epitaxy (ALE) was introduced to supplement conventional molecular beam epitaxy (MBE) and other epitaxial methods in their efforts of growing perfect semiconducting materials and their heterostructures. $A$ successful growth of CdTe and CdMnTe epilayers with the ALE method was reported previously [1-3]. Due to different growth patterns (atomic fluxes are supplied separately to get a full monoatomic cover of a surface of a growing epilayer) it was believed that smoother interfaces can be grown with the ALE method.

In this communication we compare the results of photoluminescence (PL), time-resolved PL and PL kinetics measurements of a series of CdTe/CdMnTe multi quantum well (MQW) structures. In all these structures CdMnTe barriers were grown with a conventional MBE, whereas CdTe QWs were grown with either MBE or ALE methods. In some structures the MBE grown CdTe QWs coexisted with the ALE grown to ensure that $Q W$ s were grown at similar conditions (substrate material, growth temperature, buffer layer etc.). From the present PL studies we conclude that a morphology of interfaces in CdTe QWs grown by ALE and MBE methods is quite different. 


\section{Experimental}

Four MQW structures were studied. They were grown on (100) GaAs substrate covered with thick CdTe (e.g. about $10 \mu \mathrm{m}$ thick for samples 12054a,b) and then CdMnTe (about $1 \mu \mathrm{m}$ thick for samples 12054a,b) buffer layers. The structure labelled 12054a was a reference sample. It was grown by a conventional MBE method and contained $8 \mathrm{QWs}$ (31 monolayers (ML), $20 \mathrm{ML}, 16 \mathrm{ML}, 12 \mathrm{ML}, 8 \mathrm{ML}$, $4 \mathrm{ML}, 3 \mathrm{ML}$ and $6 \mathrm{ML}$ thick - in order from a substrate to a cap layer) separated by $175 \mathrm{ML}$ thick CdMnTe ( $37.5 \%$ of $\mathrm{Mn}$ ) barriers. The sample $12054 \mathrm{~b}$ had a similar structure, but contained $4 \mathrm{QWs}(20 \mathrm{ML}, 12 \mathrm{ML}, 6 \mathrm{ML}$ and $4 \mathrm{ML}$ thick) all of them grown by ALE method. Two other samples contained QWs grown by both MBE and ALE methods. The sample 10104a contained 2 ALE-grown QWs (7 and 9 ML thick) and 1 grown by MBE (12 ML thick). The sample 12214a contained 2 QWs grown by MBE ( $4 \mathrm{ML}$ and $6 \mathrm{ML}$ thick) and $3 \mathrm{QWs}$ grown by ALE (8 ML, $12 \mathrm{ML}$ and $20 \mathrm{ML}$ thick).

PL, time-resolved PL and PL kinetics measurements were performed on the experimental setups described elsewhere [4].

\section{Results and discussion}

In Table we summarize the results of PL kinetics measurements for 4 QWs of different thickness grown by either MBE or ALE methods. These results show the implicit dependence of PL decay time on well width, similar to that observed by us previously $[4,5]$. The PL decay times at $2 \mathrm{~K}$ and for wells of the same thickness but of a different growth method are generally similar and are only slightly longer for the ALE-grown QWs. However, time-resolved PL experiments indicate more delocalized character of excitons in ALE-grown wells. For example, a larger magnitude of exciton drift towards states of a lower energy was observed during exciton decay in the ALE-grown QWs. Moreover, a stronger energy dependence (PL decay time measured within the PL band depends on detection energy) of PL decay times was observed for the ALE-grown QWs. This is also consistent with a more delocalized character of excitons in these structures [6].

Temperature dependence of PL decay times was measured. In Fig. 1 we show such dependence for the $20 \mathrm{ML}$ thick wells in the 12054a and 12054b structures. The observed temperature dependencies are slightly different for two types of MQW systems studied. PL decay time of the ALE-grown sample is weakly temperature dependent up to about $20 \mathrm{~K}$ and then starts to increase with increasing temperature. Such increase is slightly slower for the MBE-grown well, which is consistent with the results of other time-resolved PL investigations indicating a more localized character of excitons in the MBE-grown wells.

PL investigations of the ALE-grown QWs showed broad excitonic PL lines. The width of PL lines was up to few times larger than that observed for excitonic transitions in equivalent (the same width) CdTe QWs grown by MBE method. At first, it was rather a surprising result since the ALE-grown wells were assumed to have smoother interfaces and consequently narrower PL lines. Following PL kinetics measurements we relate different PL line widths to a different character of excitons in the two types of MQW systems studied. The PL lines for the 
TABLE

Excitons properties in QWs grown by either MBE or ALE methods.

\begin{tabular}{|c|c|c|c|c|}
\hline $\begin{array}{l}\text { Width of a } \\
\text { QW in MLs }\end{array}$ & $\begin{array}{l}12054 \mathrm{a} \\
\text { (MBE) }\end{array}$ & $\begin{array}{l}12054 \mathrm{~b} \\
\text { (ALE) }\end{array}$ & $\begin{array}{l}10104 a \\
\text { (MBE) }\end{array}$ & $\begin{array}{c}12214 \mathrm{a} \\
\text { (ALE/MBE) }\end{array}$ \\
\hline $4 \mathrm{ML} \mathrm{QW}$ & $315 \mathrm{ps}^{a, b}$ & $370-380 \mathrm{ps}^{a, b}$ & - & MBE-270 $\mathrm{ps}^{a, b}$ \\
\hline $6 \mathrm{ML} \mathrm{QW}$ & $250 \mathrm{ps}^{a, b}$ & $270-290 \mathrm{ps}^{a, b}$ & - & $\mathrm{MBE}-220 \mathrm{ps}^{a, b}$ \\
\hline $12 \mathrm{ML}$ QW & $240 \mathrm{ps}^{c}$ & $290 \mathrm{ps}^{a, b}$ & $215 \mathrm{ps}^{c}$ & ALE-240 ps ${ }^{a, c}$ \\
\hline 20 ML QW & $130 \mathrm{ps}$ & $125 \mathrm{ps}^{a, c}$ & - & ALE-170 $\mathrm{ps}^{a, c}$ \\
\hline
\end{tabular}

${ }^{a}$ Energy dependent decay time. The PL decay time given was measured at PL maximum.

${ }^{b}$ Strong drift of PL maximum towards lower energy.

${ }^{c}$ Weak drift of PL maximum towards lower energy.

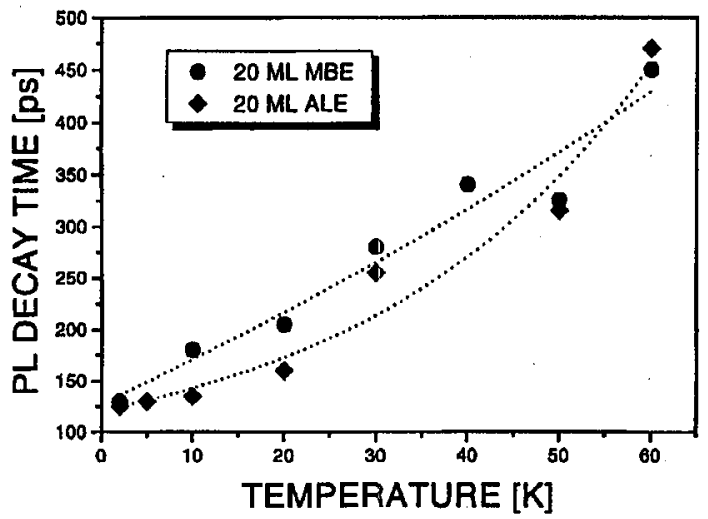

Fig. 1. Temperature dependence of PL decay times for two $20 \mathrm{ML}$ thick quantum wells grown by MBE (sample 12054a) and ALE (sample 12054b) methods.

MBE-grown wells were of a Gaussian shape indicating a dominant contribution of an inhomogeneous broadening of PL expected for either localized or quasi-localized excitons in structures with potential fluctuations in a QW plane caused by fluctuations of a well width and composition [6,7]. For the ALE-grown $20 \mathrm{ML}$ thick QW (see Fig. 2) the line shape is a mixture of Gaussian and Lorentzian (dominant contribution). In the latter case, PL line width is determined by both a statistical distribution of energies of different regions of a $\mathrm{QW}$ and by a very short excitons coherence times [6].

Time-resolved PL experiments (Fig. 2) show another difference in the PL line properties for the 20 ML thick QW grown by ALE. At 0 ps delay PL has an asymmetric shape and consists of two partly resolved PL lines separated by about $6 \mathrm{meV}$. Such energy distance is slightly larger than that expected for either free or localized and bound (neutral donor bound (DBE)) excitons [4,5]. The high energy 


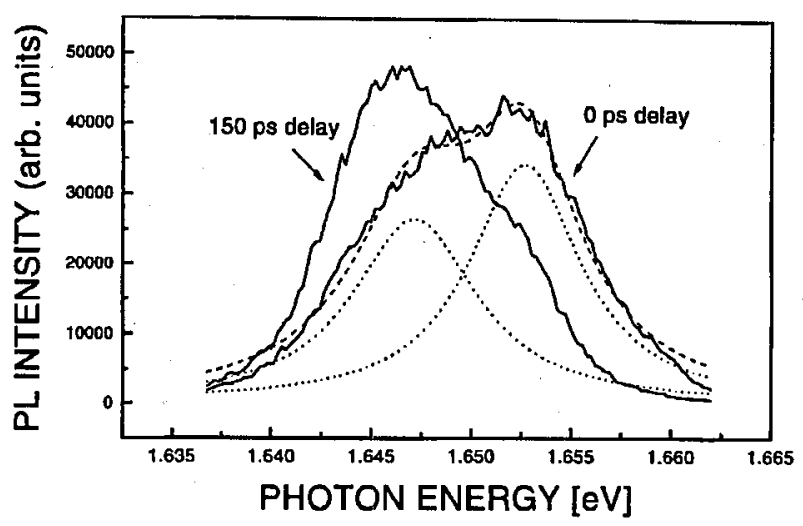

Fig. 2. Time-resolved PL spectra for the $20 \mathrm{ML}$ thick ALE-grown QW in the 12054b MQW structure measured at 0 ps delay (PL reached its maximum after 5 ps long laser pulse) and after $150 \mathrm{ps}$ of the PL decay. The dashed line represents the fit to the PL spectrum for two PL subbands (dotted lines) of Lorentzian line shape.

component of the PL rapidly disappears for longer delay times and is not observed for delays longer than $200-250 \mathrm{ps}$.

Two-line structure of QW PL was often observed for the MBE-grown $\mathrm{CdTe} / \mathrm{CdMnTe}$ samples. This structure was explained by a simultaneous observation of free (localized) and DBE excitons. The DBE PL saturates at increased excitation intensity and is dissociated once temperature is increased to about $30-40 \mathrm{~K}$. A very different intensity and temperature dependence is observed for the two PL emissions shown in Fig. 2. Both PLs are observed at increased temperature with a high energy component increasing with an increasing temperature. Moreover, we did not observe a fast decay of this PL at increased temperature. All these observations are not consistent with a free and bound exciton character of two PLs, but the results closely resemble the properties of excitons from QW regions of thickness varying by $1 \mathrm{ML}$ observed previously for growth interrupted GaAs/AlGaAs QWs [7]. We thus attribute the high energy component of the PL line to the PL emission from QW islands differing in thickness by $1 \mathrm{ML}$. If confirmed, this would be the first observation of existence of such islands in CdTe/CdMnTe MQW system.

Concluding, the described above properties of excitons in QWs indicate different morphology of interfaces in the wells grown by the ALE method. These interfaces are rougher in microscale. Moreover, the time-resolved PL studies suggest existence of QW regions of thickness differing by $1 \mathrm{ML}$ and with a planar extent large as compared to exciton radius. Excitons remain mobile in the ALE-grown wells and their line width is broadened due to homogeneous contribution caused by their short coherence times.

This work is supported in part by the Committee for Scientific Research through grant 8T 11B 01411.

\section{References}

[1] M.A. Herman, O. Jylhä, M. Pessa, J. Cryst. Growth 66, 480 (1984). 
[2] M.A. Herman, P. Juza, W. Faschinger, H. Sitter, Cryst. Res. Technol. 23, 3 (1988).

[3] M.A. Herman, M. Vulli, M. Pessa, J. Cryst. Growth 73, 403 (1985).

[4] M. Godlewski, J.P. Bergman, B. Monemar, B. Koziarska, A. Suchocki, G. Karczewski, T. Wojtowicz, J. Kossut, A. Waag, D. Hommel, Mater. Sci. Forum 196-201, 455 (1995).

[5] M. Godlewski, J.P. Bergman, B. Monemar, Lithuanian Phys. J. 35, 563 (1995).

[6] D.S. Citrin, Phys. Rev. B 47, 3832 (1993).

[7] M.A. Herman, D. Bimberg, J. Christen, J. Appl. Phys. 70, R1 (1991). 